\title{
TENDÊNCIAS NOS ÍNDICES CLIMÁTICOS E AGROCLIMÁTICOS APLICADOS À VIDEIRA NO PLANALTO SERRANO DE SANTA CATARINA
}

\author{
BACK, Álvaro José - ajb@unesc.net \\ Doutor em Engenharia - Pesquisador da Epagri - Professor do PPGCA/Unesc \\ BRUNA, Emilio Della - emilio@epagri.sc.gov.br \\ Eng. Agrônomo, MSc. Pesquisador da Epagri, Estação Experimental de Urussanga \\ FELIPETTO, João - joaofelippeto@epagri.sc.gov.br \\ Enólogo, Pesquisador da Epagri, Estação Experimental de São Joaquim
}

\begin{abstract}
RESUMO: Foram avaliadas as tendências nas séries climáticas e nos índices agroclimáticos para a cultura da videira na Região Serrana de Santa Catarina. Foram utilizados os dados diários da estação meteorológica de São Joaquim, do período de 1961 a 2010. A tendência nas séries foi avaliada pelo teste de Mann-Kendall, e para estimar a magnitude da tendência utilizou-se a declividade mediana determinada pelo teste de Theil-Sen. Os resultados mostraram tendências de aumento nas séries de temperatura mínima do ar em escala anual, bem como nas estações do ano. Para temperatura máxima do ar houve tendência significativa de diminuição nas séries de temperatura máxima anual e na temperatura máxima da primavera e verão. Com relação aos índices agrometeorológicos, foi observada uma tendência significativa de aumento da soma térmica e do índice de Frio, além de tendências não significativas nas séries do número de geadas, datas de brotação, florescimento e colheita, duração do período vegetativo, índice de Huglin. Com relação à precipitação houve tendência significativa de aumento da precipitação total anual, da precipitação no período de crescimento da cultura da videira e do número de dias com chuva igual ou superior a $20 \mathrm{~mm}$.
\end{abstract}

Palavras-chave: clima, agrometeorologia, fenologia, viticultura

TRENDS IN CLIMATIC AND AGROCLIMATIC INDICES APPLIED TO GRAPEVINE IN MOUNTAIN RANGE REGION OF SANTA CATARINA STATE

ABSTRACT: Trends in climate series and agroclimatic indices were evaluated for growing vines in the Mountain Range Region of Santa Catarina State. Daily data of the Meteorological Station of São Joaquim from 1961 to 2010 were used. The trend in the series was analyzed with the Mann-Kendall test and to estimate the magnitude of the trend it was used the median slope determined by Theil-Sen estimator. The results showed an increasing trend in series of minimum air temperature at the annual scale and also in the seasons of the year. The maximum air temperature presented a significant trend of decrease in the series of annual maximum temperature and in the maximum temperature in spring and summer. In terms of agrometeorological indices it was observed a significant trend of increase in thermal summation and in the cold index besides non-significant trends in the series in the number of frost events, date of bud break, flowering and ripening, vegetative growing season, Huglin index. In relation to precipitation there was a significant trend of increase in the annual total precipitation, in precipitation during the growth period of the vines, and in number of days with rainfall amounts equal or above than $20 \mathrm{~mm}$.

Key words: climate, agrometeorology, phenology, vitiviniculture

\section{INTRODUCÃO}

As modificações climáticas, e em especial, o aumento da temperatura do ar e alterações na distribuição e frequência de chuvas podem ter impactos na atividade vitivinícola. Os modelos climáticos apontam para aumento na 
temperatura média e também aumento da ocorrência de chuvas na Região Sul do Brasil (MARENGO et al., 2009).

Estudos que envolvem séries temporais longas de elementos meteorológicos são realizados em diferentes análises, que incluem a determinação de suas tendências (KAYANO e SANSIGOLO, 2009; BLAINET et al., 2011; SANTOS, 2011). Alexander et al., (2006) analisando dados globais extremos de temperatura do ar e precipitação pluvial, na escala diária, identificaram significativa elevação nas temperaturas noturnas em $70 \%$ das regiões analisadas.

O clima possui forte influencia na produção e qualidade da uva e na qualidade do vinho. Extremos de temperatura do ar, tanto mínimas como máximas, podem afetar de modo negativo as culturas agrícolas (STRECK et al., 2011). O aumento da temperatura pode ter impactos na produção da uva e causar modificações na área de cultivo e nas variedades cultivadas. Segundo Jones e Goodrich (2008), o aquecimento observado nos últimos 50 anos trouxe benefícios para muitas regiões vitícolas, tal como redução de geadas. Esses benefícios são mais evidentes em regiões de climas frios. Por outro lado, nas regiões vitícolas mais quentes e secas, houve necessidade de maiores investimentos para irrigação, aumentando os custos de produção.

O conhecimento dos impactos potenciais de alterações no clima sobre a viticultura é necessário para que pesquisadores e produtores tomem decisões quanto a políticas e práticas de manejo que possam minimizar os impactos negativos explorar possíveis impactos positivos.

O clima nas principais regiões produtoras de uva para vinho no mundo se caracteriza por invernos rigorosos, com muitos dias ensolarados, e baixas precipitações durante o ciclo vegetativo da videira. No Brasil, poucos são os microclimas que tem semelhanças com estas condições climáticas. Dentre as regiões do país com potencialidades, alguns microclimas, em Santa Catarina como a região de São Joaquim, no Planalto Sul Catarinense, Água Doce no Meio Oeste e Campos Novos no Planalto, tem despertado o interesse empresarial pela vitivinicultura.

A região do Planalto Serrano de Santa Catarina apresenta características climáticas bastante diferenciadas, com predominância de invernos rigorosos, primavera e verão, amenos e outono com alternância de temperaturas diurnas e noturnas e, baixo índice pluviométrico na época de maturação e de colheita. Embora as perspectivas para expansão do cultivo de uvas viníferas nesta região sejam boas, é importante destacar que são poucas as informações técnicocientíficas disponíveis, notadamente, no que diz respeito ao comportamento vitícola-enológico das variedades nos diferentes microclimas potenciais do Estado.

Esse trabalho teve como objetivo avaliar as tendências nos dados climáticos e nos índices agroclimáticos relacionados com a viticultura da região do Planalto Serrano de Santa Catarina, SC. 


\section{MATERIAL E METODOS}

Foram utilizadas as séries históricas diárias de temperatura máxima, mínima e média do ar e precipitação pluvial do período de 1961 a 2010, registrados na estação meteorológica de São Joaquim (latitude $28^{\circ} 17^{\prime} 34^{\prime \prime}$ S, longitude 490 55' 56" W, altitude de 1388 metros). Na figura 1 pode-se visualizara região do Planalto Serrano de Santa Catarina e a localização do município de São Joaquim.

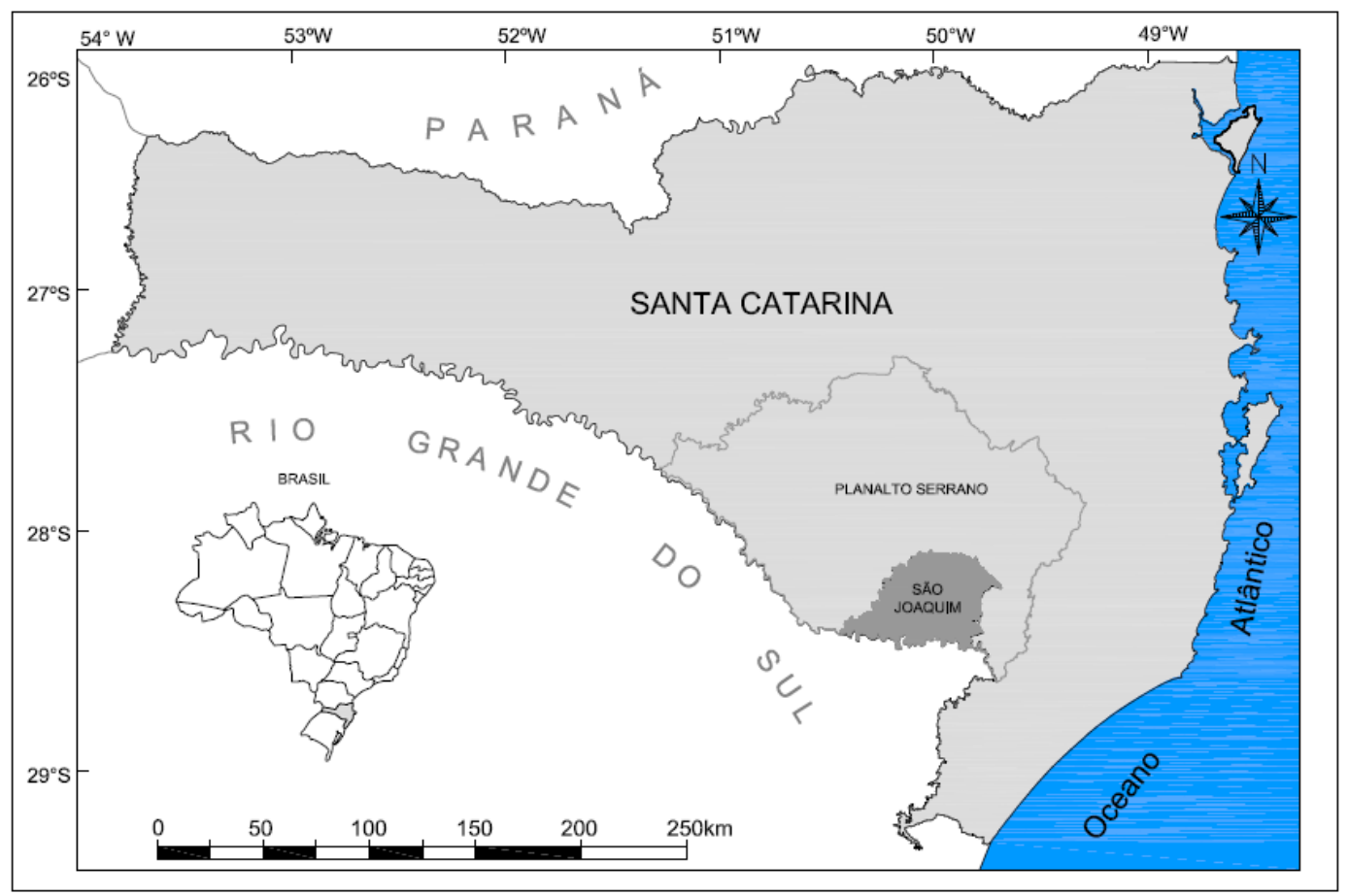

Figura 1. Area do Planalto Serrano e Santa Catarina

O clima da região, de acordo com o sistema de classificação climática de Koeppen, é classificado como mesotermico úmido e verão ameno (Cfb). A temperatura média normal anual da zona agroecológica do Planalto Serrano de São Joaquim varia de 11,4 a $14,3^{\circ} \mathrm{C}$. A temperatura média normal das máximas varia de 16,9 a $19,4^{\circ} \mathrm{C}$ e das mínimas de 7,6 a $9,2^{\circ} \mathrm{C}$. A precipitação pluviométrica total normal anual varia de 1450 a $1650 \mathrm{~mm}$, com o total anual de dias chuvosos de em torno de 135 dias.

Foram calculados os indices climáticos definidos pela Equipe de Especialista em Detecção, Monitoramento e Índices de Mudanças Climáticas (Expert Team on Climate Change Detection, Monitoringand Índices-ETCCDMI) que se encontram descritos em Sensoy et al., (2007), e índices agroclimáticos aplicados a videira (Tabela 1). Estes índices foram calculados em escala anual, por estação do ano e também na estação de crescimento da cultura da videira, considerado de setembro a fevereiro. 
O potencial impacto das mudanças climáticas na viticultura foi detectado pela aplicação de índices agroclimáticos tais como: número de dias com geada (FD); graus dias na estação de crescimento (GDD) relacionados com a data de início das fases fenológicas mais importantes (brotação, florescimento e colheita); a soma térmica com $10^{\circ} \mathrm{C}$ de temperatura basal de setembro a fevereiro (STA10); o índice de Frio (IF); o índice de Huglin (IH). Segundo Orlandini et al., (2009), estes índices são bons indicadores da interação entre mudanças no clima e as necessidades fisiológicas das plantas.

Tabela 1. Índices climáticos e agroclimáticos usados e suas definições

\begin{tabular}{|c|c|c|}
\hline Índice & Unidade & Descrição \\
\hline TX a & ${ }^{\circ} \mathrm{C}$ & Média da temperatura máxima diária (anual) \\
\hline TX sp & ${ }^{\circ} \mathrm{C}$ & Média da temperatura máxima diária (primavera) \\
\hline TX s & ${ }^{\circ} \mathrm{C}$ & Média da temperatura máxima diária (verão) \\
\hline TX au & ${ }^{\circ} \mathrm{C}$ & Média da temperatura máxima diária (outono) \\
\hline TX w & ${ }^{\circ} \mathrm{C}$ & Média da temperatura máxima diária (inverno) \\
\hline TN a & ${ }^{\circ} \mathrm{C}$ & Média da temperatura mínima diária (anual) \\
\hline TN sp & ${ }^{\circ} \mathrm{C}$ & Média da temperatura mínima diária (primavera) \\
\hline TN S & ${ }^{\circ} \mathrm{C}$ & Média da temperatura mínima diária (verão) \\
\hline TN au & ${ }^{\circ} \mathrm{C}$ & Média da temperatura mínima diária (outono) \\
\hline TN w & ${ }^{\circ} \mathrm{C}$ & Média da temperatura mínima diária (inverno) \\
\hline TN90p & dias & $\begin{array}{l}\text { Número de dias com temperatura mínima acima do } \\
\text { percentil } 90(1961-1990)\end{array}$ \\
\hline TX90p & dias & $\begin{array}{l}\text { Número de dias com temperatura máxima acima do } \\
\text { percentil } 90(1961-1990)\end{array}$ \\
\hline T a & ${ }^{\circ} \mathrm{C}$ & Media da temperatura media diária (anual) \\
\hline TXX a & ${ }^{\circ} \mathrm{C}$ & Valor máximo anual de temperatura máxima diária \\
\hline TNx a & ${ }^{\circ} \mathrm{C}$ & Valor mínimo anual de temperatura mínima diária \\
\hline FD & Dias & $\begin{array}{l}\text { Numero de dias o ano } \mathrm{cm} \text { temperatura mínima } \\
\text { inferior } 0^{\circ} \mathrm{C}\end{array}$ \\
\hline VGSO & Dias & $\begin{array}{l}\text { Duração do período vegetativo(limite } 0^{\circ} \mathrm{C} \text { ): Numero } \\
\text { de dias entre a última e a primeira geada de cada ano }\end{array}$ \\
\hline GDDb & Dias & $\begin{array}{l}\text { Data da brotação: é o dia do ano quando a soma } \\
\text { térmica (temperatura base } 10^{\circ} \mathrm{C} \text { ) atinge o valor } \\
\text { específico }\end{array}$ \\
\hline GDDf & Dias & $\begin{array}{l}\text { Data de florescimento: é o dia do ano quando a soma } \\
\text { térmica (temperatura base } 10^{\circ} \mathrm{C} \text { ) atinge o valor } \\
\text { específico }\end{array}$ \\
\hline GDDr & Dias & $\begin{array}{l}\text { Data da colheita: é o dia do ano quando a soma } \\
\text { térmica (temperatura base } 10^{\circ} \mathrm{C} \text { ) atinge o valor } \\
\text { específico }\end{array}$ \\
\hline HI & graus & $\begin{array}{l}\text { Índice de Huglin: soma diária da média entre a } \\
\text { temperatura máxima e média calculada durante a } \\
\text { estação de crescimento (setembro a fevereiro) } \\
\text { multiplicado pelo coeficiente de latitude }\end{array}$ \\
\hline IF & ${ }^{\circ} \mathrm{C}$ & $\begin{array}{l}\text { Índice de Frio: temperatura mínima do mês } \\
\text { antecedente a colheita (fevereiro) }\end{array}$ \\
\hline
\end{tabular}




\begin{tabular}{|c|c|c|}
\hline STA10 & ${ }^{\circ} \mathrm{C}$ & $\begin{array}{l}\text { Soma térmica (temperatura base } 10^{\circ} \mathrm{C} \text { ): soma da } \\
\text { temperatura media diária acima de } 10^{\circ} \mathrm{C} \text { no período } \\
\text { de crescimento (setembro a fevereiro) }\end{array}$ \\
\hline PRCPTOT & $\mathrm{mm}$ & Precipitação total anual (PR $\geq 1,0$ mm) \\
\hline PRCPgs & $\mathrm{mm}$ & $\begin{array}{l}\text { Precipitação total (PR } \geq 1,0 \mathrm{~mm} \text { ) durante a estação } \\
\text { de crescimento (Setembro to Fevereiro) }\end{array}$ \\
\hline $\mathrm{R} 20$ & dias & Número de dias no ano com chuva superior a $20 \mathrm{~mm}$ \\
\hline
\end{tabular}

Para a definição das datas de brotação (GDDb) florescimento (GDDf) e colheita (GDDr) foram consideradas as datas em que a soma térmica alcança os valores de 110,400 e 1500 graus dias, respectivamente.

A equação utilizada para determinar a soma térmica foi:

$$
\mathrm{ST} \mathrm{A10}=\sum_{\mathrm{i}=1}^{\mathrm{n}}\left(\mathrm{T}_{\text {med }}-10\right)
$$

em que:

STA10 - soma das temperaturas médias superiores a $10^{\circ} \mathrm{C}$ (graus dias);

$\mathrm{T}_{\text {med }}$ - temperatura média diária $\left({ }^{\circ} \mathrm{C}\right)$, calculada como:

$$
\mathrm{T}_{\text {med }}=\frac{\mathrm{T}_{\mathrm{x}}+\mathrm{T}_{\mathrm{n}}}{2}
$$

em que:

$\mathrm{T}_{\mathrm{x}}$ - temperatura máxima diária $\left({ }^{\circ} \mathrm{C}\right)$

$\mathrm{T}_{\mathrm{n}}$ - temperatura mínima diária $\left({ }^{\circ} \mathrm{C}\right)$

O Índice de Huglin foi calculado segundo a fórmula:

$$
\mathrm{HI}=\sum \frac{\left(\mathrm{T}_{\text {med }}-\mathrm{T}_{\mathrm{b}}\right)+\left(\mathrm{T}_{\mathrm{x}}-\mathrm{T}_{\mathrm{b}}\right)}{2} \mathrm{k}
$$

em que:

$\mathrm{HI}$ - índice de Huglin $\left({ }^{\circ} \mathrm{C}\right)$

$\mathrm{Tb}$ - temperatura de base $\left(10^{\circ} \mathrm{C}\right)$

$K$ - coeficiente fixo $(k=1)$, com um valor específico para a latitude da região em estudo (BORGHEZAN et al., 2011)

Com relação à precipitação foram considerados os índices de precipitação total anual (PRCPTOT a), precipitação na estação de crescimento (PRCPgs) e o número de dias de chuva superior a $20 \mathrm{~mm}$ (R20). Nos cálculos destes índices somente foram considerados as chuvas iguais ou superiores a $1,0 \mathrm{~mm}$.

Para avaliar a tendência de mudanças climáticas foi utilizado o teste não paramétrico de Mann-Kendall (BURNET et al., 2004), sendo a estatística MannKendall, $\mathrm{S}$ dada por:

$$
S=\sum_{j=1}^{N-1} \sum_{i=j+1}^{N} \operatorname{sgn}\left(x_{i}-x_{j}\right)
$$

em que:

$\mathrm{X}$ - valores das séries temporais para todos $\mathrm{i}, \mathrm{j} \leq \mathrm{N}$

i e $\mathrm{j}$ - as sequências dos dados

$\mathrm{N}$ - comprimento da série

em que: 
$\operatorname{sgn}(\theta)=\left\{\begin{array}{c}1 \text { se } \theta>0 \\ 0 \operatorname{se} \theta=0 \\ -1 \text { se } \theta<0\end{array}\right\}$

Para variável aleatória independente distribuída identicamente (sem tendência) a média e a variância de $\mathrm{S}$ são dadas por:

$$
\begin{aligned}
& E(S)=0 \\
& \operatorname{Var}(S)=\frac{N(N-1)(2 N+5)-\sum_{t}^{N} t(t-1)(2 t+5)}{18}
\end{aligned}
$$

em que:

t - extensão a qualquer empate

Para o teste bicaudal, ao nível de significância a, rejeita-se Ho se o teste estatístico padronizado, $Z<Z$ a/2 ou $Z>Z$ a/2, em que a estatística normalizada $Z$ de Mann-Kendall segue a distribuição normal com média zero e variância um. $\mathrm{O}$ valor de $\mathrm{Z}$ é calculado por:

$$
Z=\left\{\begin{array}{l}
\frac{S-1}{\sqrt{\operatorname{var}(S)}} \text { se } S>0 \\
0 \text { se } S=0 \\
\frac{S+1}{\sqrt{\operatorname{var}(S)}} \text { se } S<0
\end{array}\right\}
$$

O teste de Mann-Kendall não fornece estimativa da magnitude da tendência. Para obter esta estimativa foi utilizado o teste não paramétrico de Theil-Sen (HELSEL e HIRSCH, 2002), em que a estimativa robusta da inclinação ( $\beta$ ) é obtida por:

$\beta=\operatorname{median} a\left(\frac{Y_{j}-Y_{i}}{t_{j}-t_{i}}\right)$, para todos $i>j$,

em que:

$\beta$ - mediana da inclinação obtida para todas as possíveis combinações de dois pontos da série temporal

Y - a variável testada para tendência

$\mathrm{T}$ - o tempo

\section{RESULTADOS E DISCUSSÃO}

$\mathrm{Na}$ Tabela 2 constam os valores de " $Z$ " do teste de Mann-Kendall com a respectiva significância (valor " $p$ "). Também consta a declividade mediana $(\beta)$ com os respectivos intervalos de confiança obtido pelo teste Theil-Sen. Com relação aos dados de temperatura máxima constata-se a tendência na redução dos valores de temperatura máxima, com tendência significativa para a temperatura máxima na primavera ( $T X \mathrm{sp}$ ) e no verão ( $\mathrm{TX} \mathrm{s}$ ). A declividade média $(\beta)$ na primavera e verão foi de $-0,0143^{\circ} \mathrm{C} /$ ano e $-0,0167^{\circ} \mathrm{C} / \mathrm{ano}$, 
respectivamente, o que equivalem à redução nos valores de temperatura da ordem de $1,5^{\circ} \mathrm{C} /$ século.

Para os valores de temperatura mínima observa-se tendência significativa de aumento na temperatura mínima anual (TN a) e nos valores de temperatura mínima de cada estação, exceto na primavera (TN $s p)(p=0,071)$. A declividade média da temperatura mínima anual foi de $1,74^{\circ} \mathrm{C} /$ século, com valores superiores a $2,0^{\circ} \mathrm{C} /$ século nas estações de verão, outono e inverno. $\mathrm{A}$ temperatura média anual ( $\mathrm{T}$ a) apresentou declividade média de $0,0071^{\circ} \mathrm{C}$, no entanto a tendência não foi significativa $(p=0,146)$.

Com relação às temperaturas extremas não foi constatada tendência significativa nas séries de temperaturas máximas absolutas anuais ( $T X x$ a) e mínimas absolutas anuais (TNx a). No entanto, os valores de TX90p apresentaram tendência de redução $(\beta=-0,2391 \mathrm{~d} / \mathrm{ano})$ e TN90p apresentou tendência significativa de aumento $(\beta=0,5000 \mathrm{~d} / \mathrm{ano})$, o que equivale ao aumento de cinco noites quentes a cada dez anos.

De acordo com a análise dos dados de temperatura máxima e mínima, é possível inferir que existe uma tendência significativa de diminuição das temperaturas máximas diárias. Por outro lado, o estudo demonstrou, também, um aumento nas temperaturas mínimas diárias, o que leva a uma redução na amplitude térmica diária. Embora a maioria dos trabalhos verificados na literatura tenha mostrado tendências significativas de aumento nas temperaturas máximas e mínimas, também existem estudos que demonstram um aumento mais acentuado da temperatura mínima em relação á temperatura máxima (DAI et al., 1997). Isto é denominado de aquecimento assimétrico, implicando na diminuição da amplitude térmica. Segundo Nemaniet et al., (2001), o aquecimento global tem como consequência o aumento da evaporação e do conteúdo de vapor na atmosfera, aumentando, assim, a nebulosidade e a incidência de chuvas. Tal aquecimento assimétrico na temperatura foi largamente relatado por Easterling et al., (1999).

Os resultados obtidos no presente trabalho estão de acordo vários estudos realizados nas Regiões Sul e Sudeste do Brasil. Blain et al., (2009) indicaram tendências significativas de elevação em séries anuais de temperatura média máxima e especialmente média mínima do ar. Blain (2011) analisando as séries mensais de temperatura máxima e mínima do Estado de São Paulo conclui que as alterações de ordem climática observadas nas séries de TX são bastante inferiores às observadas nas séries de TN. Blain e Lulu (2011), não identificaram tendências significativas nas séries de máximas e mínimas absolutas em dados coletados a partir de seis estações meteorológicas distribuídas no Estado de São Paulo. Dufek e Ambrizzi (2006) indicaram que neste Estado, há indícios de tendências para uma condição atmosférica mais quente. Os autores descrevem que particularmente para as regiões norte e central do Estado, essa elevação associa-se a diminuição de dias frios nos anos de 1990 a 2002, sendo uma condição mais severa no período de inverno. 
Tabela 2. Resultados dos testes estatísticos da tendência dos índices climáticos aplicados ao cultivo da videira em São Joaquim, SC

\begin{tabular}{lccccccc}
\hline & \multicolumn{1}{c}{ Teste Mann-Kendall } & \multicolumn{5}{c}{ Teste Theil-Sen } \\
\cline { 2 - 8 } Índice & $\mathrm{S}$ & $\mathrm{Z}$ & Valor p & Mediana & $\beta$ & $\beta$ inferior & $\beta$ superior \\
\hline TX a & -263 & $-1,955$ & 0,051 & 18,9 & $-0,0095$ & $-0,0183$ & 0,0000 \\
TX sp & -265 & $-1,970$ & 0,049 & 18,7 & $-0,0143$ & $-0,0276$ & 0,0000 \\
TX s & -44 & $-3,007$ & 0,003 & 22,8 & $-0,0167$ & $-0,0273$ & $-0,0056$ \\
TX au & -122 & $-0,903$ & 0,367 & 18,8 & $-0,0056$ & $-0,0217$ & 0,0091 \\
TX w & -67 & $-0,492$ & 0,623 & 15,2 & $-0,0045$ & $-0,0200$ & 0,0128 \\
TN a & 458 & 3,409 & 0,001 & 9,6 & 0,0174 & 0,0077 & 0,0286 \\
TN SP & 243 & 1,805 & 0,071 & 8,9 & 0,0133 & 0,0000 & 0,0281 \\
TN s & 313 & 2,328 & 0,020 & 12,9 & 0,0206 & 0,0028 & 0,0367 \\
TN au & 376 & 2,774 & 0,006 & 10,1 & 0,0200 & 0,0069 & 0,0333 \\
TN w & 291 & 2,164 & 0,030 & 6,1 & 0,0235 & 0,0000 & 0,0406 \\
IF & 449 & 3,342 & 0,001 & 12,4 & 0,0318 & 0,0134 & 0,0486 \\
T a & 196 & 1,455 & 0,146 & 14,1 & 0,0071 & 0,0000 & 0,0143 \\
TXx a & -215 & $-1,597$ & 0,110 & 28,0 & $-0,0118$ & $-0,0308$ & 0,0000 \\
TNx a & 138 & 1,022 & 0,307 & $-5,3$ & 0,0205 & $-0,0111$ & 0,0464 \\
TX90 & -388 & $-2,887$ & 0,004 & 32,5 & $-0,2391$ & $-0,4000$ & $-0,0714$ \\
TN90 & 478 & 3,559 & 0,000 & 40,0 & 0,5000 & 0,2566 & 0,7500 \\
FD & -175 & $-1,298$ & 0,194 & 23,5 & $-0,1000$ & $-0,2500$ & 0,0476 \\
VGS0 & 121 & 0,895 & 0,371 & 215,5 & 0,3158 & $-0,3255$ & 0,9160 \\
STA10 & 382 & 2,842 & 0,004 & 1966,8 & 2,7238 & 0,8406 & 4,7330 \\
GDDb & -73 & $-0,537$ & 0,591 & 280,0 & $-0,0571$ & $-0,2996$ & 0,1875 \\
GDDf & -88 & $-0,649$ & 0,516 & 314,0 & $-0,0667$ & $-0,3077$ & 0,1429 \\
GDDc & -98 & $-0,724$ & 0,469 & 69,0 & $-0,0714$ & $-0,2941$ & 0,1138 \\
HI & -181 & $-1,307$ & 0,191 & 1659,25 & $-1,2083$ & $-2,8436$ & 0,4941 \\
PRPCTOT & 279 & 2,325 & 0,020 & 1687,9 & 9,4000 & 1,7352 & 17,3600 \\
P gs & 337 & 2,811 & 0,005 & 933,6 & 7,0000 & 2,1060 & 11,2200 \\
R20 & 250 & 2,083 & 0,037 & 27,5 & 0,2000 & 0,0000 & 0,3810 \\
\hline
\end{tabular}

As diferenças sazonais nas tendências dos dados de temperatura também foram constatadas por outros autores. Segundo Marengo et al., (2010) na Região Sul do Brasil a tendência na temperatura a partir da década de 60 indica mais forte aquecimento na temperatura mínima do que máxima, principalmente no inverno (JJA), resultando diminuição da amplitude térmica diária. Estes autores também observaram redução significativa do número de dias muito frios, mas não encontraram mudanças significativas na frequência de dias muitos quentes. Vincent et al., (2000), analisando dados diários de temperaturas extremas da América do Sul entre 1960 e 2000, observaram que não houve tendência nos índices baseados na temperatura máxima. No entanto, constataram mudanças significativas na porcentagem de noites quentes e tendência significativa de 
diminuição na porcentagem de noites frias em várias estações. Segundo esses autores, as alterações nas séries temporais das temperaturas diurnas tiveram significância e coerência espacial consideravelmente inferior às verificadas na temperatura noturna. Kayano e Sansigolo (2009) detectaram tendências significativas em índices associados à temperatura mínima atmosférica (escalas anual e sazonal), com taxas de elevação próximas as apontadas pelos relatórios do IPCC. Entretanto, para índices associados às temperaturas máximas atmosféricas estes autores descrevem tendências climáticas apenas para o período do verão. Essa tendência foi de diminuição na temperatura ou resfriamento da ordem de $-0,6^{\circ} \mathrm{C}$ a cada 100 anos.

O número de geadas anuais (FD) apresentou mediana de 23,5 geadas anuais, com o intervalo entre o último e o primeiro evento de geada no ano (VGSO) com mediana de 215,5 dias. Não foram encontradas tendências significativas nestes índices agrometeorológicos. Da mesma, forma não foi constatada tendência significativa nas séries de GDDb, GDDfe GDDr.

O índice de frio (IF) é utilizado como indicador das condições de temperatura noturna durante a maturação. Os dados mostram que o IF mediano foi de 12,4 ${ }^{\circ} \mathrm{C}$, com tendência significativa de aumento $\left(\beta=0,0318^{\circ} \mathrm{C} / \mathrm{ano}\right)$. De acordo com Conceição e Tonietto (2005) o valor de IF de $12,4^{\circ} \mathrm{C}$ caracteriza a região como classe "de noites frias $(12<$ IF $\leq 14)$ ". O IF serve como indicador das características potenciais das regiões em relação aos metabólitos secundários (polifenóis, aroma, cor) nas uvas e vinhos, e é relacionado com a qualidade da uva, principalmente com aroma e coloração. Em condições nictotérmicas quentes de maturação, a tendência é de verificar perda de aromas e coloração relativamente fraca. As condições nictotérmicas frias de maturação são essencialmente favoráveis á coloração e aos aromas das uvas.

A soma térmica mostrou tendência de aumento com declividade mediana de $2,7238^{\circ} \mathrm{C} d / a n o$, e deve-se principalmente a elevação das temperaturas mínimas. De acordo com Santos et al., (2009), um clima com dias quentes e noites quentes, produz vinho com estilo diferente, na comparação com uma região ainda com a mesma soma térmica, mas com dias quentes e noites frias. Quando temperaturas noturnas se aproximam de $10^{\circ} \mathrm{C}$ a baga cessa a maturação. Quando a temperatura noturna eleva-se acima daquele patamar, a maturação continua durante a noite. Assumindo-se que a temperatura diurna aproxima-se do ótimo para maturação, a menor amplitude térmica diária produz a maturação mais rápida que climas com maior amplitude térmica diária. $\mathrm{Na}$ prática, para uma determinada variedade, micro climas com menor amplitude térmica diária geralmente maturam o fruto com maior teor de sólidos solúveis totais, na comparação com climas com maior amplitude térmica e maiores índices de nebulosidade e com similar soma térmica.

O índice de Huglin (HI) não apresentou tendência significativa. O HI é usado para classificação de regiões vitícolas, para caracterizar a adaptação do cultivar às condições climáticas, para caracterização de terroirs. Também é utilizado para monitoramento do desenvolvimento fisiológico e bioquímico da uva, e para definição do estágio fenológico e data de colheita (TONIETTO e CARBONNEAU, 
2004). O valor da mediana do $\mathrm{HI}$ foi de $1659,25{ }^{\circ} \mathrm{C}$, classificado como "Frio $(1500<\mathrm{HI} \leq 1800)^{\prime \prime}$.

Segundo Tonietto e Carbonneau (2004), na classe de clima vitícola Frio o potencial heliotérmico possibilita maturar uma gama relativamente ampla de uvas, brancas ou tintas incluindo a Cabernet Franc. Este índice tem considerável implicação para a viticultura, pois as temperaturas noturnas são importantes para o desenvolvimento do aroma do fruto, e do $\mathrm{pH}$ do suco em termos de degradação do ácido málico sob condições de elevadas temperaturas. Índices de noites frescas podem ser indiretamente relacionados à intensidade do aroma do vinho e coloração do suco de variedades rosadas (Carbonneau et al., 2007). Carey e Bonnardot (2004) mostraram que existem relações entre o índice de Huglin e o aroma do vinho.

Com relação à precipitação os índices mostraram aumento significativo na chuva anual (PRPCTOT) ( $\beta=9,4 \mathrm{~mm} / \mathrm{ano})$, na chuva durante a estação de crescimento (PRPC gs) $(\beta=7,0 \mathrm{~mm} / \mathrm{ano})$. Com relação aos dias de chuva acima e $20 \mathrm{~mm}$ (R20) observa-se tendência de aumento ( $\beta=0,20$ chuvas /ano).

A cultura da videira tem sua produção e qualidade afetadas tanto pelo excesso como pelo déficit hídrico. A água é um elemento fundamental para o crescimento vegetativo e reprodutivo da videira, para o seu funcionamento fisiológico e bioquímico. A água também é um fator determinante no rendimento e qualidade das uvas e nas características dos vinhos (DELOIRE et al., 2005). O excesso hídrico combinado com temperaturas elevadas torna a cultura da videira muito suscetível a doenças fúngicas e pragas. Uma seca moderada na maturação favorece a qualidade dos frutos da videira. Segundo Back e Della Bruna (2009), a videira pode ser cultivada sem irrigação a partir de uma precipitação anual de 500 a $600 \mathrm{~mm}$ por ano. Elevada precipitação durante o período de florescimento causa falhas na frutificação durante a maturação, causa apodrecimento dos frutos e perda de qualidade. A precipitação mediana durante a estação de crescimento foi de $933,6 \mathrm{~mm}$, o que indica que em geral há excesso de precipitação na região de São Joaquim.

A constatação de tendência de aumento da chuva anual bem como da chuva durante o período vegetativo implica em preocupação com relação à qualidade da uva e dificuldade na realização dos tratos culturais. Espera-se maior incidência de doenças pelo aumento da chuva. Segundo Pedro Junior et al,. (1999), a aplicação de fungicidas após a chuva de $20 \mathrm{~mm}$ controla satisfatoriamente as doenças fúngicas na videira, principalmente a antracnose nos cachos, folhas e ramos. Bardin et al., (2010) usaram o critério da pulverização após a chuva de $20 \mathrm{~mm}$ para determinar o risco de ocorrência de doenças fúngicas na videira Niágara Rosada no estado de São Paulo. Dessa forma, pode-se inferir pelo índice R20 no período vegetativo um aumento do número de pulverizações contra doenças fúngicas, com consequência de aumento dos custos e diminuição da qualidade dos frutos. 
4. CONCLUSÕES

1. Foi observado redução da temperatura máxima, com tendência significativa nas séries de temperatura máxima na primavera (TXsp) e verão (TXs).

2. Foi observada tendência significativa de aumento nas séries de temperatura mínima anual (TNa) e da temperatura mínimanas estações, com exceção da primavera (TNsp).

3. Não foi observado tendência significativas nas séries de temperatura máxima absoluta (TXXa) e temperatura mínima absoluta anual (TNxa), no entanto valores de TX90p apresentam tendência de diminuição e os valores de TN90p apresentaram tendência de aumento.

4. Não foi constatada tendência significativa nos índices agroclimáticos de número de geadas (FD), data de brotação (GDDb), florescimento (GDDf) e colheita (GDDr) e no índice de Huglin (HI).

5. Foi observada tendência significativa de aumento do índice de Frio (IF) e da soma térmica (STA10).

6. Com relação a precipitação os índices mostraram aumento significativo na precipitação total (PRPCTOT), na precipitação durante a fase de desenvolvimento da videira (PRPgs) e no número de dias com chuva acima de $20 \mathrm{~mm}$ (R20).

\section{REFERÊNCIAS BIBLIOGRÁFICAS}

ALEXANDER, L. V.; ZHANG, X.; PETERSON, T. C.; CAESAR, J.; GLEASON, B.; KLEIN TANK, A. M. G.; HAYLOCK, M.; COLLINS, D.; TREWIN, B.; RAHIMZADEH, F.; TAGIPOUR, A.; RUPA KUMAR, K.; REVEDEKAR, J.; GRIFFITHS, G.; VICENT, L.; STEPHENSON, D.B.; BACK, A. J.; DELLA BRUNA, E. Demanda hídrica e necessidade de irrigação da videira para Urussanga, SC. Agropecuária Catarinense, v.22, p.76-81, 2009.

BARDIN, L.; PEDRO JUNIOR, M. J.; MORAES, J. F. L. Risco climático de ocorrência de doenças fúngicas na videira Niagara Rosada na região do pólo turístico do circuito das frutas do Estado de São Paulo. Bragantia, v.69, p.1019-1026, 2010.

BLAIN, G. C; LULU, J. Valores anuais absolutas de séries de temperatura máxima e mínima do ar no Estado de São Paulo: Tendências, periodicidades e probabilidades associadas. Revista Brasileira de Meteorologia, v.26, p.29-40, 2011.

BLAIN, G. C. Considerações estatísticas relativas a seis séries mensais de temperatura do ar da Secretaria de Agricultura e Abastecimento do Estado de São Paulo. Revista Brasileira de Meteorologia, v.26, p.279-296, 2011.

BLAIN, G. C.; PICOLE, M. C. A.; LULU, J. Análises estatísticas das tendências de elevação nas séries de temperatura mínima do ar no Estado de São Paulo. Bragantia, v.68, p.807-815, 2009.

BORGHEZAN, M.; GAVIOLI, O.; PIT, F. A.; SILCA, A. L. Comportamento vegetativo e produtivo da videira e composição da uva em São Joaquim, Santa Catarina. Pesquisa Agropecuária Brasileira, v.46, p.398-405, 2011.

CARBONNEAU, A.; DELOIRE, A.; JAILLARD, B. La vigne: Physiologie, terroir, culture. Paris: Dunod, 2007. 441p.

CAREY, V.; BONNARDOT, V. A viticultural perspective of meso-scale atmospheric modelling in the Bottelaryberg-Simonsberg-Helderberg wine growing area (South Africa).

Bulletin de l'Office International de la Vigne et du Vin, v.77, p.20-46, 2004.

CONCEIÇÃO, M. A. F.; TONIETTO, J. Climatic potential for wine grape production in the tropical north region of Minas Gerais State, Brazil. Revista Brasileira de Fruticultura, v.37, p.404-407, 2005.

DAI, A.; DEL GENIO, A. D.; FUNG, I. Y. Clouds, precipitation and temperature range. Nature, v.386, p.665-666, 1997. 
DELOIRE, A.; OJEDA, H.; ZEBIC, O.; BERNARD, N.; HUNTER, J. J.;CARBONNEAU, A. Influence de l'état hydrique de la vigne sur le style de vin. Progrès Agricole et Viticolev.122, p.455-462, 2005.

DUFEK, A. S., AMBRIZZI, T. Precipitation variability in São Paulo State, Brazil. Theoretical and Applied Climatology, v.93, p.167-178, 2008

EASTERLING, D. R.; DIAZ, H. F. DOUGLAS, A.V.; HOGG, W. D.; KUNKEL, K.E.; ROGERS, J. C.; WILKINSON, J.F. Long-term observations for monitoring extremes in the Americas. Climatic Change, v.42, p.285-308, 1999.

HELSEL, D. R.; HIRSCH, R. M. Statistical methods in water resources -hydrologic analysis and interpretation: Techniques of Water-Resources investigations of the U. S. Washington: Geological Survey, chap. A3, book 4, 2002. 510p.

JONES, G. V.; GOODRICH, G. B. Influence of climate variability on wine regions in the western USA and on wine quality in Napa Valley. Climate Research, v.35, p.241-254, 2009.

KAYANO, M. T.; SANSIGOLO, C. Interannual to decadal variations of precipitation and daily maximum and minimum temperature in Southern Brazil. Theoretical and Applied Climatology, v.97, p.81-90, 2009.

MARENGO, J. A.; RUSTICUCCI, M.; PENALBA, O.; RENOM, M. An intercoparison of observed and simulated extreme rainfall and temperature events during the last half of the twentieth century: part 2:historical trends. Climatic Change, v.98, p.509-529, 2010.

MARENGO, J. A.; JONES, R.; ALVES, L. M.; VALVERDE, M. C. Future change of temperature and precipitation extremes in South America derived from PRECIS regional climate modeling system. International Journal of Climatology, v.29, p.2241-2255, 2009.

NEMANI, R. R.; WHITE, M. A.; CAYAN, D. R.; JONES, G. V.; RUNNING, S. W.; COUGHLAN, J. C.; PETERSON, D. L. Asymmetric warming over coastal California and its impact on the premium wine industry. Climate Research, v.19, p.25-34, 2001.

ORLANDINI, S.; DI STEFANO, V.; LUCCHESINI, J. L. ; PUGLISI, A. BARTOLINI, G. Current trends of agroclimatic indices applied to grapevine in Tuscany (Central Italy). Idójaras, v.113, p.69-78, 2009.

PEDRO JUNIOR, J. J.; PEZZOPANE, J. R. M.; MARTINS, E. P. Uso da precipitação pluvial para previsão de épocas de pulverização visando controle de doenças fúngicas na videira "Niagara Rosada'. Revista Brasileira de Agrometeorologia, v.7, p.107-111, 1999.

SANTOS, A. O.; ROLIM, G. DE S.; HERNANDES, J. L.; PEDRO JÚNIOR, M. J. A maturação fisiológica da videira em São Paulo: Comentários sobre as safras de verão e de inverno na média altitude paulista. 2009. Artigo em Hypertexto. <http://www.infobibos.com/Artigos/2009_3/maturacao/index.htm>. 26 Fev. 2013.

SANTOS, C. A. C. Trends in indices for extremes in daily air temperature over Utah, USA. Revista Brasileira de Meteorologia, v.26, p.19-28, 2011.

STRECK, N. A.; GABRIEL, L. F.; BURIOL, G. A.; HEDWEIN, A. B. PAULA, G. M. Variabilidade interdecadal na série secular de temperatura do ar em Santa Maria, RS. Pesquisa Agropecuária Brasileira, v.46, p.781-790, 2011.

SENSOY, S.; PETERSON, T. C.; ALEXANDER, L. V.; ZHANG, X. Enhancing middle east climate change monitoring and indexes. Bulletin of American Meteorological Society,n.88, v8, p.1249-1254, 2007.

TONIETTO, J.; CARBONNEAU, A. A multicriteria climatic classification system for grapegrowing regions worldwide. Agricultural and Forest Meteorology, v.124, p.81-97, 2004.

VICENT, L. A.; PETERSON, T. C. ; BARROS, V. R.; MARINO, M. B. ; RUSTICUCCI, M.; CARRASCO, G.; RAMIREZ, E.; ALVES, L. M.; AMBRIZZI, T. BERLATO, M. A.; GRIMM, A. M.; MARENGO, J. A.; MOLION, L.; MONCUNILL, D. F.; REBELLO, E.; ANUNCIAÇÃO, Y. M. T.; QUINTANA, J.; SANTOS, J. L.; BAEZ, J.; CORONEL, G.; GARCIA, J.; TREBEJO, I.; BIDEGAIN, M.; HAYLOCK, M. R. KAROLY, D. Observed trends in indices of Daily temperatures extremes in South America 1960-2000. Journal of Climate, v.18, p.5011-5023, 2005. 